\title{
Multifocal Visual Evoked Potentials Reveal Normal Optic Nerve Projections in Human Carriers of Oculocutaneous Albinism Type 1a
}

\author{
Michael B. Hoffmann, ${ }^{1}$ Barbara Wolynski, ${ }^{1}$ Synke Meltendorf, ${ }^{1}$ \\ Wolfgang Behrens-Baumann, ${ }^{1}$ and Barbara Käsmann-Kellner ${ }^{2}$
}

\begin{abstract}
Purpose. In albinism, part of the temporal retina projects abnormally to the contralateral hemisphere. A residual misprojection is also evident in feline carriers that are heterozygous for tyrosinase-related albinism. This study was conducted to test whether such residual abnormalities can also be identified in human carriers of oculocutaneous tyrosinase-related albinism (OCA1a).
\end{abstract}

METHODS. In eight carriers heterozygous for OCA1a and in eight age- and sex-matched control subjects, monocular pattern-reversal and -onset multifocal visual evoked potentials (mfVEPs) were recorded at 60 locations comprising a visual field of $44^{\circ}$ diameter (VERIS 5.01; EDI, San Mateo, CA). For each eye and each stimulus location, interhemispheric difference potentials were calculated and correlated with each other, to assess the lateralization of the responses: positive and negative correlations indicate lateralizations on the same or opposite hemispheres, respectively. Misrouted optic nerves are expected to yield negative interocular correlations. The analysis also allowed for the assessment of the sensitivity and specificity of the detection of projection abnormalities.

REsults. No significant differences were obtained for the distributions of the interocular correlation coefficients of controls and carriers. Consequently, no local representation abnormalities were observed in the group of OCA1a carriers. For pattern-reversal and -onset stimulation, an assessment of the control data yielded similar specificity (97.9\% and 94.6\%) and sensitivity (74.4\% and $74.8 \%$ ) estimates for the detection of projection abnormalities.

Concuusions. The absence of evidence for projection abnormalities in human OCA1a carriers contrasts with the previously reported evidence for abnormalities in cat-carriers of tyrosinase-related albinism. This discrepancy suggests that animal models of albinism may not provide a match to human albinism. (Invest Ophthalmol Vis Sci. 2008;49:2756-2764) DOI:10.1167/iovs.07-1461

From the ${ }^{1}$ Universitäts-Augenklinik, Magdeburg, Germany; and the ${ }^{2}$ Klinik für Augenheilkunde, Universitätsklinikum des Saarlandes, Homburg, Germany. $1 / 2$

Supported by German Research Council Grant DFG HO-2002/4-

Submitted for publication November 15, 2007; revised January 29, 2008; accepted April 9, 2008

Disclosure: M.B. Hoffmann, None; B. Wolynski, None; S. Meltendorf, None; W. Behrens-Baumann, None; B. Käsmann-Kellner, None

The publication costs of this article were defrayed in part by page charge payment. This article must therefore be marked "advertise ment" in accordance with 18 U.S.C. $\$ 1734$ solely to indicate this fact.

Corresponding author: Michael B. Hoffmann, Universitäts-Augenklinik, Visual Processing Laboratory, Leipziger Strrasse 44, 39120 Magdeburg, Germany; michael.hoffmann@med.ovgu.de.
Tn humans, the nasal retina projects to the contralateral and 1 the temporal retina to the ipsilateral hemisphere. As a consequence, the line of decussation that divides crossed from uncrossed fibers normally coincides with the vertical meridian through the fovea. This normal projection of visual fibers from the retina is substantially altered in albinism, where the line of decussation is shifted into the temporal retina, such that a great number of fibers from the temporal retina cross the midline and project contralaterally. ${ }^{1-7}$ As a consequence, the visual cortex receives abnormal input. ${ }^{8}$ Remarkably, it is still not understood by which mechanisms the disturbance of the melanin synthesis mediates the misrouting of the optic nerves during embryonic development. Recent studies in humans even suggest that an albinotic phenotype may not be an essential prerequisite for the misrouting to occur. ${ }^{9-11}$

Albinism is an inherited disorder that can be caused by a variety of mutations on several genes. ${ }^{12,13}$ While ocular albinism (OA1) is associated with an X-chromosome-linked inheritance, oculocutaneous albinism (OCA) is associated with an autosomal-recessive inheritance. Small projection abnormalities have been demonstrated in cats that are normally pigmented heterozygous carriers of OCA. More specifically, the cats carry a gene coding for a dysfunctional tyrosinase ${ }^{14,15}$ and are thus homologous to the human OCA1a-type albinism. These reports of cat models of albinism have spurred several hypotheses concerning human carriers of albinism: They indicate that misrouted optic nerves may be evident in otherwise phenotypically inconspicuous human carriers of a dysfunctional tyrosinase gene. Consequently, they imply that misrouting of the optic nerves may have a much higher incidence in humans than originally assumed. Finally, they suggest that misrouting of the optic nerves may serve as a marker of the carrier status such that carriers of albinism can be identified simply by the detection of misrouted optic nerves.

Studies testing human carriers of albinism for misrouted optic nerves are needed to test whether these cat findings are also evident in humans. Visual evoked potentials (VEPs) are a valuable tool for identifying misrouted optic nerves in human albinism. ${ }^{16}$ As reviewed in Table 1 , human carriers have so far predominantly been examined with conventional visual evoked potential paradigms. Almost all these studies failed to reveal any projection abnormalities (the single exception reported VEP signatures that are not a definitive indicator of misrouting ${ }^{23}$ ), which may either be due to the actual absence or to the strongly localized nature of potential abnormalities. In the latter case, conventional VEPs may not be sensitive enough to detect local abnormalities. As conventional VEPs pool responses across a large expanse of visual field, small local representation abnormalities are likely to be masked by the residual normal representation. In contrast, multifocal VEPs enable us to record cortical responses from a great number of distinct visual field locations within a short time interval. ${ }^{26-29}$ They can therefore support the identification of small representation abnormalities and have indeed recently been demonstrated to assist the detection of misrouted optic nerves. ${ }^{25}$ In 
TABLE 1. Studies on Projection Abnormalities in Carriers of Albinism

\begin{tabular}{|c|c|c|c|c|}
\hline Species & hz Albinism-type & Method & Misrouting* & Study \\
\hline Cat & hhOCA1a & sce \& h & $5 / 5\left(15^{\circ}\right)$ & Leventhal et al. ${ }^{14}$ \\
\hline \multirow[t]{2}{*}{ Cat } & hhOCA1a & sce $\& \mathrm{~h}$ & $2 / 2\left(19^{\circ}\right)$ & Ault et al. ${ }^{15}$ \\
\hline & Siamese cats & sce $\& \mathrm{~h}$ & $0 / 2$ & Ault et al. ${ }^{15}$ \\
\hline Human & Not specified & VEP & $0 / 10$ & Creel et al. ${ }^{2}$ \\
\hline Human & Not specified & VEP & $0 / 9$ & Apkarian et al. ${ }^{17}$ \\
\hline Human & No OCA1a & VEP & $0 / 15$ & Castle et al. ${ }^{18}$ \\
\hline Human & OCA & VEP & $0 / 2$ & Shallo-Hoffmann and Apkarian ${ }^{19}$ \\
\hline Human & OA1 & VEP & $0 / 1$ & Apkarian $^{20}$ \\
\hline Human & Not specified & VEP & $0 / 10$ & Fitzgerald and Cibis ${ }^{21}$ \\
\hline Human & Not specified & VEP & $0 / 3$ & Russel-Eggitt et al. ${ }^{22}$ \\
\hline Human & OA1 & VEP & $2 / 2$ & Rudolph et al. ${ }^{23}$ \\
\hline Human & OA1 & VEMF & $0 / 1$ & Lauronen et al. ${ }^{24}$ \\
\hline Human & OA1 & $\mathrm{mfVEP}$ & $0 / 5$ & Hoffmann et al. ${ }^{25}$ \\
\hline
\end{tabular}

hz, heterozygous for; hh, homologous to human; sce, single cell electrophysiology; h, histology; VEP, visual evoked potentials; VEMF, visual evoked magnetic fields.

* Number of individuals with misrouting from total (if applicable, maximum extent of misrouting is given in parentheses).

that study, misrouted optic nerves were evident in subjects with albinism, but not in carriers of ocular albinism (i.e., of albinism type OA1). This discrepancy to cat study findings, however, is not a conclusive indicator of interspecies differences, as it may be due to the different genotype investigated. The appropriate human equivalent to the feline carriers with residual misrouting are human carriers of OCA1a. Therefore, we investigated whether residual misprojections of the optic nerves are a general trait of human carriers of OCA1a.

\section{Materials AND Methods}

\section{Subjects}

Participating in the study were eight obligatory carriers heterozygous for OCA1a (age range, 33-38 years; further details in Table 2). A ninth carrier had to be excluded due to heavy contamination of the mfVEP recordings with $\alpha$-rhythms. Eight sex- and age-matched ( \pm 3 years) subjects (age range, 31-40 years) with monocular visual acuity $\geq 1.0$ (Freiburg Acuity Test ${ }^{31}$ ) participated in the study as control subjects. All subjects gave their informed written consent before the study. The procedures adhered to the tenets of the Declaration of Helsinki, and the protocol was approved by the ethics committee of the University of Magdeburg, Germany.

\section{VEP Recordings}

Rationale of VEP Detection of Albinotic Misrouting of the Optic Nerves. In albinism, each eye projects predominantly to its contralateral hemisphere. Monocular stimulation of the central visual field is therefore expected to elicit greater VEPs on the hemisphere contralateral to the stimulated eye than on the ipsilateral hemisphere. As a consequence, the polarity of the interhemispheric VEP difference is inverted for left compared with right eye stimulation in subjects with albinism. In contrast, in control subjects the polarity does not depend on the eye stimulated. ${ }^{17}$ Supplementing this paradigm with a correlation analysis simplifies the approach and enhances its objectivity. In albinism, the interhemispheric activation differences obtained between right and left eye stimulation are likely to be negatively correlated, because of the polarity inversion of the traces. In contrast, in control subjects (i.e., in the absence of such a polarity inversion), the activation differences are likely to be positively correlated. ${ }^{9,32-34}$ This correlation approach supports an objective analysis even of small signals. To obtain a greater spatial resolution when sampling the visual field for representation abnormalities, the VEP paradigm for the detection of misrouted optic nerves can be combined with the multifocal stimulation technique. ${ }^{25}$

Stimulation. A visual evoked potential recording system (VERIS 5.01.10X; EDI, San Mateo, CA) was used for stimulus delivery and electrophysiological recordings. While supported by a chin rest, sub-

TABLE 2. Characteristics of Carriers Heterozygous for OCA1a

\begin{tabular}{|c|c|c|c|c|c|c|c|c|c|c|c|c|c|}
\hline \multirow[b]{2}{*}{ Subject } & \multirow[b]{2}{*}{ Sex } & \multirow[b]{2}{*}{ Age } & \multicolumn{2}{|c|}{$\begin{array}{l}\text { Visual } \\
\text { Acuity }\end{array}$} & \multirow{2}{*}{$\begin{array}{l}\text { Visual } \\
\text { Field }\end{array}$} & \multicolumn{3}{|c|}{ Stereo Tests } & \multicolumn{2}{|c|}{ Pigmentation } & \multirow{2}{*}{$\begin{array}{c}\text { Macular } \\
\text { Hypoplasia }\end{array}$} & \multirow[b]{2}{*}{ Papilla } & \multirow[b]{2}{*}{ Nyst. } \\
\hline & & & OD & OS & & Lang & Titmus & TNO & Fundus & Iris & & & \\
\hline 1 & M & 33 & 1.2 & 1.3 & $\mathbf{N}$ & + & $40^{\prime \prime}$ & $30^{\prime \prime}$ & $\mathbf{N}$ & $\mathbf{N}$ & - & $\mathbf{N}$ & - \\
\hline 2 & $\mathrm{~F}$ & 36 & 1.0 & 1.4 & $*$ & + & $40^{\prime \prime}$ & $30^{\prime \prime}$ & $\mathbf{N}$ & $\mathbf{N}$ & - & $\ddagger$ & - \\
\hline 3 & M & 38 & 1.5 & 1.6 & $\mathbf{N}$ & + & $40^{\prime \prime}$ & $15^{\prime \prime}$ & $\mathbf{N}$ & $\mathbf{N}$ & - & $\mathbf{N}$ & - \\
\hline 4 & M & 37 & 0.8 & 1.0 & $\mathrm{~N}$ & - & - & - & $\mathbf{N}$ & $\mathbf{N}$ & - & $\mathrm{N}$ & - \\
\hline 5 & $\mathrm{~F}$ & 36 & 0.8 & 1.0 & $\mathbf{N}$ & - & $800^{\prime \prime}$ & - & $\mathbf{N}$ & $\mathbf{N}$ & - & $\mathrm{N}$ & - \\
\hline 6 & $\mathrm{~F}$ & 35 & 1.2 & 1.3 & $*$ & + & $40^{\prime \prime}$ & $60^{\prime \prime}$ & $\dagger$ & $\mathbf{N}$ & - & $\int$ & - \\
\hline 7 & M & 36 & 0.8 & 1.2 & $\mathbf{N}$ & - & - & - & $\mathrm{N}$ & $\mathbf{N}$ & - & $\mathrm{N}$ & - \\
\hline 8 & M & 35 & 1.3 & 1.8 & $\mathbf{N}$ & + & $40^{\prime \prime}$ & $15^{\prime \prime}$ & $\mathrm{N}$ & $\mathrm{N}$ & - & $\mathbf{N}$ & - \\
\hline
\end{tabular}

Pigmentation iris, iris translucency assessed with slit lamp; Nyst., nystagmus; n, normal; -, negative or absent; +, positive or present.

* Slightly reduced visual field sensitivity in the peripheral upper hemifield in both eyes.

† Slight retinal hypopigmentation in the periphery (grade 1, according to Käsmann-Kellner et al. ${ }^{12}$ and Schmitz et al. ${ }^{30}$ ).

$\neq$ Bilateral small and oblique papilla.

$\S$ Bilateral excavation of the papilla. 
jects viewed the stimuli that were presented at a distance of $34 \mathrm{~cm}$ on a computer monitor driven at a frame rate of $75 \mathrm{~Hz}$. They were requested to fixate the center of a central black cross that spanned, analogous with our previous studies, the entire stimulus and thus served as a highly salient fixation target. The stimulus display, a circular dartboard pattern (diameter, $44^{\circ}$; mean luminance, $64 \mathrm{~cd} / \mathrm{m}^{2}$; and contrast, 97\%), was subdivided into individual fields, each comprising a checkerboard of $4 \times 4$ checks. The radial extent of the fields was scaled with eccentricity from $1.5^{\circ}$ in the center to $7^{\circ}$ in the periphery. The fields were stimulated independently with an m-sequence. Msequences consist of a pseudorandom succession of states 0 and 1. For pattern-reversal stimulation, these two states were represented by two contrast-inverted checkerboard fields. As the minimal duration of one state lasted one frame (i.e., $13.3 \mathrm{~ms}$ ), the maximum and mean patternreversal rates were 75 and 37.5 reversals per second, respectively. For pattern onset, stimulation state 0 was represented by a succession of two gray fields, whereas state 1 was represented by a succession of checkerboard pattern and gray. As the minimal duration of one state lasted two frames (i.e., $26.6 \mathrm{~ms}$ ), the maximum and mean pattern-onset rates were 37.5 and 18.75 pattern onsets per second, respectively. Thus, the states lasted twice as long for pattern-onset stimulation as for pattern-reversal stimulation, as pattern-onset/offset comprised a frame of pattern plus a frame of uniform gray for the elemental state. Therefore, a single block of pattern-reversal stimulation lasting 7 minutes consisted of an m-sequence with $2^{15}-1$ elements, whereas a single block of pattern-onset stimulation for the same duration consisted of an m-sequence with $2^{14}-1$ elements. The blocks were divided into 16 overlapping segments each lasting approximately 27 seconds.

Procedure. Recording sessions were conducted in a dimly lit room and lasted $\sim 3$ hours, including preparation and breaks. A recording session comprised eight 7 -minute-blocks $(2 \times 2 \times 2$ : two repetitions, two eyes, and two stimulation modes, i.e., pattern-reversal and pattern-onset). The blocks were presented in a balanced, interleaved sequence (extended a-b-b-a scheme). Multifocal VEPs (mfVEPs) were recorded with six gold-cup electrodes referenced to the inion, placed 4 and $8 \mathrm{~cm}$ to the left and right of the location, $1 \mathrm{~cm}$ above the inion (lateral occipital sites), and $5 \mathrm{~cm}$ left and right of the location, and $7 \mathrm{~cm}$ above the inion (lateral parietal sites). The EEG was amplified with a physiological amplifier (Grass-Telefactor, West Warwick, RI), bandpass filtered (low- and high-frequency cutoffs 3 and $70 \mathrm{~Hz}$ ), and digitized at $1200 \mathrm{~Hz}$.

mfVEP Analysis. First- and second-order kernels for patternonset and -reversal stimulation were extracted with the visual evoked potential recording system (VERIS 5.01; EDI). The spatial-smoothing and artifact-rejection features available in the system were not used. All subsequent analyses were performed with a commercial programming package (IGOR 5.0; WaveMetrics, Inc., Lake Oswego, OR). The traces were digitally low-pass filtered with a high-frequency cutoff of $30 \mathrm{~Hz}$. To assess the lateralization of the responses, we calculated the difference potentials between each of the three electrodes on one hemisphere and its corresponding electrode on the other hemisphere. These difference potentials entered the further analysis.

To assess signal presence, we evaluated the signal-to-noise ratio (SNR), as described by Zhang et al. ${ }^{35}$ using a mean noise-window SNR. First, the records from the two blocks for each stimulus were averaged. Then the SNR for each $i$ th sector (of the 60 total sectors) of subject $j$ was defined as

$$
\mathrm{SNR}_{\mathrm{ij}}=\mathrm{RMS}_{\mathrm{ij}}(45-150 \mathrm{~ms}) /\left[\Sigma_{\mathrm{i}} \mathrm{RMS}_{\mathrm{ij}}(325-430 \mathrm{~ms}) / n\right]-1 .
$$

The denominator in equation 1 is the average of the individual root mean square (RMS) values of 60 sectors in the noise window (325- 430 ms after stimulus onset). An estimate of false-positive rates was obtained from the distribution of SNR values in the noise window for each $i$ th sector, $j$ th subject, $m$ th electrode pair, and $q$ th condition, according to Hood et al. ${ }^{36}$ :

$$
\mathrm{SNR}_{\mathrm{ijmq}}=\mathrm{RMS}_{\mathrm{ijmq}}(325-430 \mathrm{~ms}) /\left[\Sigma_{\mathrm{i}} \mathrm{RMS}_{\mathrm{ijmq}}(325-430 \mathrm{~ms}) / n\right]-1 .
$$

Thus, we calculated $i \times j \times m \times q$ SNRs (i.e., 5,760 values; $i=60$ locations; $j=16$ subjects; $m=$ three electrode pairs; $q=$ two conditions; left and right eye stimulation). An analysis of the distribution of these SNRs showed that the probability that SNRs $\geq 0.75$ would be part of the noise distribution was lower than $3.5 \%$. We therefore applied an SNR threshold of 0.75 to exclude silent visual field locations (i.e., those without recordable signals, from our analyses). Thus, we included visual field locations with suprathreshold responses (i.e., with recordable signals), which we will refer to as responsive locations. In our quantitative analyses we compared two stimulus conditions (i.e., left and right eye stimulation). Each stimulus location was required to evoke suprathreshold responses in at least one of the two conditions to enter the analysis (logical "OR" operator). Thus, a bias of the results toward one of these two conditions because of the thresholding procedure was avoided (e.g., an "AND" operator would lead to an exclusion of stimulus locations that are suppressed below the SNR threshold in only one of the two stimulus conditions and would, as a consequence, cause an underestimation of possible interocular differences of the responses).

For each visual field location, we selected the difference potential for the pair of electrodes on opposing hemispheres that yielded the greatest SNR during stimulation of either eye for further analysis. ${ }^{36}$ This method ensured that the same electrode pair was selected for left and right eye stimulation. Next, the difference VEPs obtained for each eye were correlated with each other to obtain Pearson's correlation coefficient ( $r=-1$ to 1 ). For this correlation, we used the signal time window $(45-150 \mathrm{~ms})$. The correlation allows for the distinction of normal and abnormal projections of the optic nerves: Positively correlated traces indicate that both eyes project to the same cortical regions, while negatively correlated traces indicate that both eyes project to opposite hemispheres. ${ }^{32,34}$ It should be noted that the correlation approach is a more objective approach than a single peak analysis and therefore allows one to deal with small signal amplitudes.

\section{Statistics}

Differences in the correlation coefficient frequencies of the two subject groups were tested for significance with Student's $t$-tests and subsequently corrected for multiple testing (four tests for each correlation threshold and each visual field region of interest) using a sequential Bonferroni correction. ${ }^{37}$

\section{Results}

To assess the interhemispheric activation differences, we calculated the difference traces of the mfVEPs recorded at symmetrical electrode sites on the left and right scalp were calculated. An example of such difference traces from a control subject to pattern-reversal stimulation is given in Figure 1A. Responses for left and right eye stimulation are juxtaposed (for a particular visual field location, traces were derived from the same electrode pairs for left and right eye stimulation, as described in the Methods section). The signals were characterized by a great variability of signal strength and shape across the visual field, which is a well-known feature of mfVEPs and is related to the cortical convolution. In contrast, the responses obtained for the two eyes for a particular visual field location resembled each other, which indicates that they were similarly affected by the cortical convolution. As a consequence, a correlation of these traces yielded predominantly positive correlation coefficients as is demonstrated in Figure 1B. It is common to depict mfVEPs recorded at central superior sites referenced to central inferior sites (e.g., Oz versus Iz). It should be noted that we used a different scheme from the usual one, to extract interhemispheric activation differences. We depict 
Figure 1. Comparison of right and left eye mfVEP responses to patternreversal stimulation in a control subject. Traces and symbols are arranged according to the spatial layout of the visual field locations that evoked them; traces and symbols from different eccentricities are arranged in an equidistant manner, whereas the actual stimulus layout is approximately $\mathrm{m}$-scaled. (A) Interhemispheric mfVEP difference traces after right (black traces) and left eye stimulation (gray traces). The responses varied across the visual field, for a particular visual field location; however, similar traces were obtained from both eyes. (B) Correlation of interhemispheric mfVEP differences to stimulation of the left and right eyes. The strength of the correlation is indicated by the diameter of the circles: The diameter of the circles scale linearly with the absolute correlation coefficient obtained, the resulting diameters for correlation coefficients of \pm 1.0 and \pm 0.5 are given at bottom right. The sign of the correlation is indicated by the style of the circles: Solid symbols: positive correlation (i.e., a normal projection pattern); open symbols: negative correlation (i.e., an abnormal projection pattern). Gray crosses: visual field locations with subthreshold responses. Arrow: visual field location with a negative interocular correlation.
A
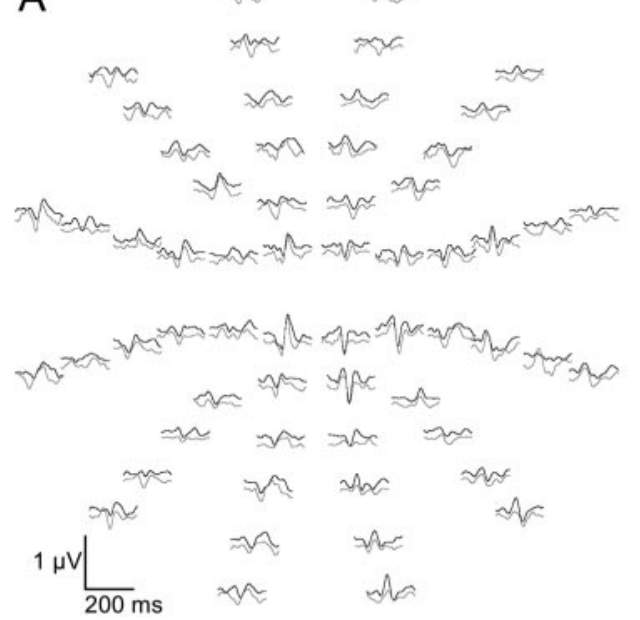

B
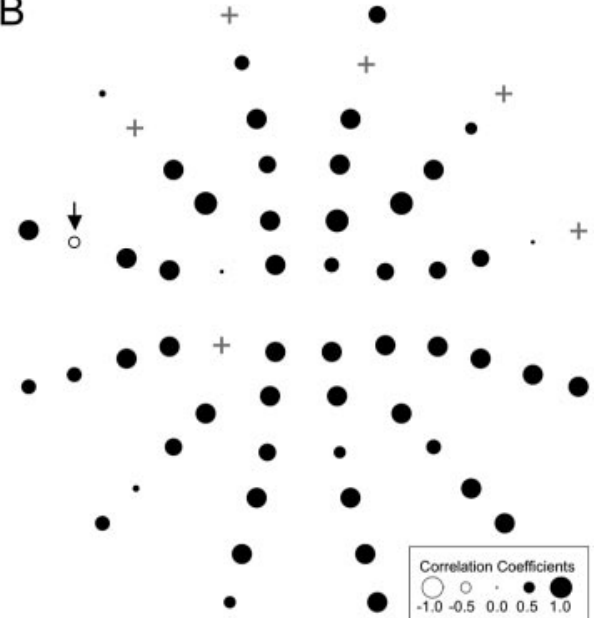

differences in mfVEPs recorded at symmetrical electrode sites on opposing hemispheres. Consequently, instead of a polarity inversion of mfVEPs from opposite sides of the horizontal meridian, as would be expected for mfVEPs recorded from central recording pairs, there is a tendency of traces on opposite sides of the vertical meridian to have inverted polarities for the interhemispheric mfVEP differences shown in Figure 1A. This polarity inversion reflects the sensitivity of these bipolar recordings to interhemispheric activation differences. If the responses to stimulation of the right and left eye are represented on opposite hemispheres, as is the case for some extent of the visual field in subjects with albinism, the correlation of the responses obtained for the two eyes will no longer be positive, but instead will be negative. ${ }^{25}$

For a quantitative assessment of the data we compared the results for the two groups. We obtained similar percentages of visual field locations yielding suprathreshold SNRs for controls and carriers for pattern reversal responses (mean \pm SD): $83.1 \%$ $\pm 11.5 \%$ and $79.6 \% \pm 15.1 \%$, respectively, and for pattern onset responses, $66.3 \% \pm 18.2 \%$ and $61.9 \% \pm 13.3 \%$, respectively. The difference between pattern-reversal and -onset responses is probably associated with the lower number of responses collected for pattern-onset stimulation, resulting in elevated noise levels and consequently, lower SNRs (see the Methods section).

First, we provide the results of pattern-reversal stimulation (Figs. 2, 3, and 4), followed by a concise overview (Fig. 5) that also covers the pattern-onset responses. To assess, whether in carriers of OCA1a, there are some visual field locations that follow the abnormal representation pattern evident in patients with albinism, the frequency distribution as a function of the interocular correlation coefficients was determined for the control and the carrier groups. The results of pattern-reversal stimulation are depicted as a histogram in Figure 2. Similar distributions of correlation coefficients were evident for both groups. Cumulative histograms were computed from these distributions, which allows for a straightforward assessment of the percentage of visual field locations with negative correlation coefficients. Again, similar distributions were evident (Fig. 3A). To assess whether the tendency of the carrier group data toward slightly more negative correlation coefficients may be caused by a minority of subjects with extreme distributions, the cumulative histograms are given for the individual controls and for the carriers in Figure 3B (for pattern-reversal stimulation). Although there was a tendency for some carriers to have an elevated number of correlation coefficients $<0.0$, only a few cases with clearly negative correlation coefficients (i.e., $<-0.5$ ) were evident. This result indicates that there may be a tendency for the responses obtained in the two eyes to correlate slightly less in the carriers than in the controls. However, there was no clear indication that the responses from the two eyes were represented on opposite hemispheres; such a representation would be associated with an elevated number of correlation coefficients that fall below a value of $-0.5 .^{25}$ Local projection abnormalities, smaller than the spatial resolution of the mfVEP, cannot be fully excluded. Alternatively, however, the tendency of the carrier group data toward slightly more negative correlation coefficients may be related to its tendency to slightly lower SNRs. Interocular mfVEP correlations are correlated with the mfVEP-SNRs (present study $[P<0.001]$ and reports by Hood et al. $\left.{ }^{38,39}\right)$.

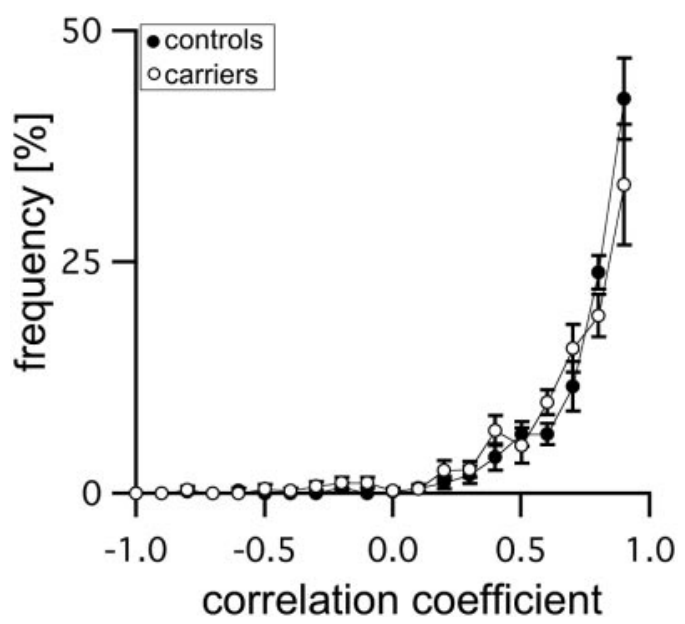

Figure 2. Frequency distribution of the correlation coefficients obtained in controls and carriers for interocular correlations of interhemispheric mfVEP differences to pattern-reversal stimulation. Similar distributions were obtained for both groups. 
A
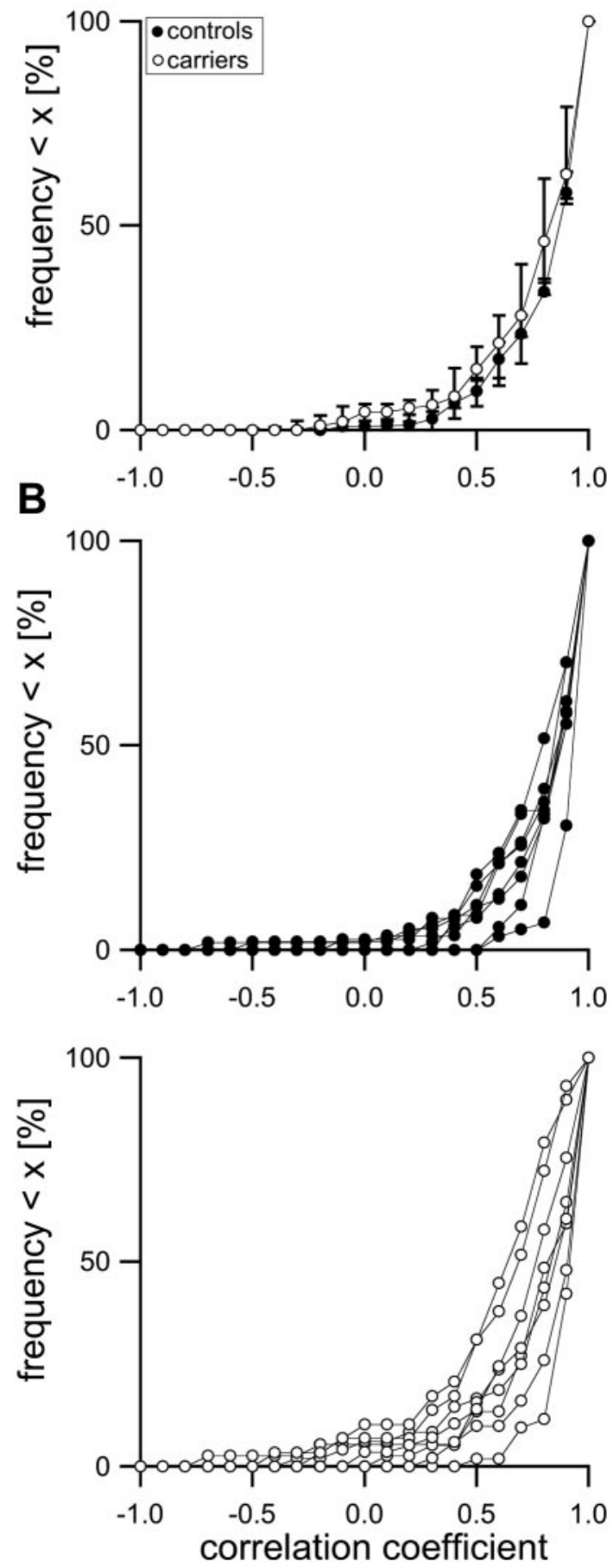

Figure 3. Cumulative histograms of the correlation coefficients obtained in controls and carriers for interocular correlations of interhemispheric mfVEP differences in response to pattern-reversal stimulation. (A) Group analysis. Similar distributions for the two groups were evident. (B) Individual histograms for controls (top) and carriers (bottom).

For a further assessment of the individual responses, the original difference pattern-reversal mfVEP traces are given in Figure 4 for three extreme cases of carriers. For carrier A, no negative correlation coefficients were obtained, indicating a particularly normal representation. In contrast, for carriers B and $\mathrm{C}$, comparatively high frequencies of negative correlation coefficients were obtained. Still, as for the control data shown in Figure 1, there was only one location in each subject with a clear indication of inverted-difference traces for the left and right eyes (Figs. 4B, 4C, see arrows).

For a detailed quantitative comparison of the distributions of the correlation coefficients, the frequency of correlation coefficients below $0.5,0.0$, and -0.5 in the control group were compared with those in the carrier group. There were no significant differences of the interocular correlations between the two groups (Fig. 5), similar to both pattern-reversal and -onset mfVEPs, which were also included in this analysis. Furthermore, this finding not only held for an analysis covering the entire visual field (Fig. 5, top), but it was also evident if the above analysis is confined to the stimulus locations within the central $\pm 10^{\circ}$ of visual field (Fig. 5, bottom). This finding is of particular importance, as visual pathway abnormalities associated with albinism have a tendency to be confined to the visual field center. ${ }^{34,40}$ Finally, the sensitivity of the mfVEP approach to detecting representations on opposite hemispheres, as they are typical in interocular response comparisons in albinism, was assessed in analogy to a previous report. ${ }^{25}$ In albinism, negative interocular correlations indicate visual field representations on opposite hemispheres. In controls, response representations on opposite hemispheres are, due to the normal projection pattern, expected after stimulation in opposite (i.e., left and right) hemifields. As a consequence, responses evoked by stimulation in opposite hemifields are expected to correlate negatively. The percentage of actually negative correlation coefficients obtained after interhemifield correlations will therefore indicate the sensitivity of the approach to detect responses on opposite hemispheres. Therefore, the responses from controls to stimulation at visual field locations that are mirrored symmetrically along the central vertical meridian correlated. The resulting correlation coefficient frequencies are shown in Figure 5 (interhemifield correlations). It is evident that a median of 74.4\% (upper quartile: $77.2 \%$; lower quartile: $71.3 \%$ ) of the correlation coefficients were negative. Consequently, for a threshold correlation coefficient of 0.0 , the sensitivity of the detection of responses on opposite hemispheres (i.e., the hit rate) was $\sim 74.4 \%$, which is in agreement with the previous report. ${ }^{25}$ Similar values were obtained for patternonset stimulation (median: $74.8 \%$; upper quartile: $77.4 \%$; and lower quartile: $70.9 \%$ ). The observed sensitivity of $\sim 75 \%$ may be associated with interhemispheric asymmetries of the cortical morphology and with reduced amplitudes in some visual field locations. The specificity of the approach for a threshold correlation coefficient of 0.0 is indicated by the percentage of positive correlation coefficients obtained for interocular correlation, which was 97.9 (upper quartile: 98.7; lower quartile: 97.0) for pattern reversal and 94.6 (upper quartile: 97.7; lower quartile: 94.57) for pattern onset.

\section{Discussion}

\section{Misrouting in Human Carriers of Albinism?}

In animal studies, projection abnormalities have been reported in subjects that are heterozygous normally pigmented carriers of albinism. ${ }^{14,15}$ Although the extent of these abnormalities was smaller than in subjects with albinism, it could still be considerable and reach by more than $15^{\circ}$ into the temporal retina. In the present study we used the mfVEP to investigate the human homologue to the feline carriers of the above studies (i.e., OCA1a carriers). With this approach, which allows sampling of the visual field for potential representation abnormalities with a higher spatial resolution than conven- 
A

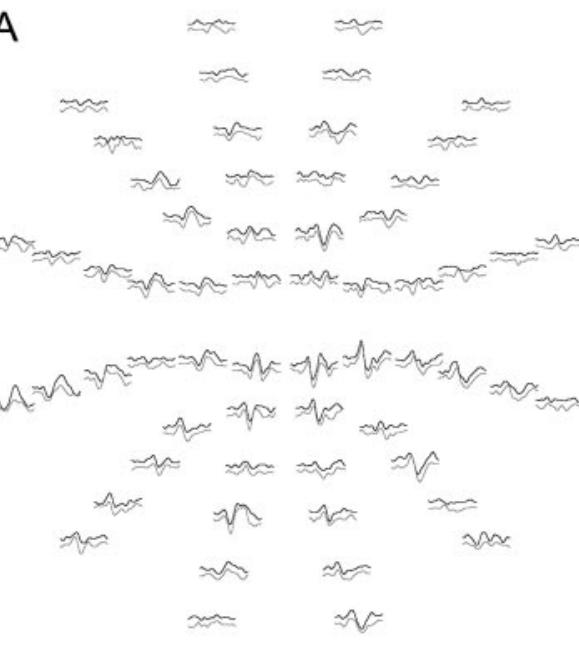

B
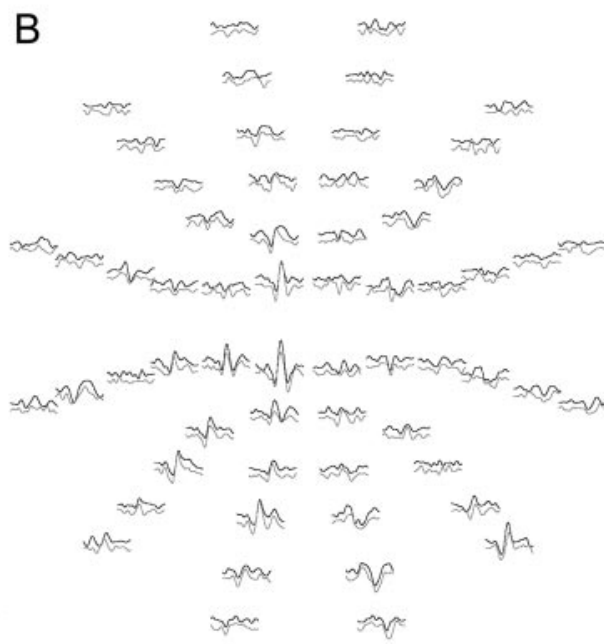

C

FIGURE 4. Comparison of right and left eye mfVEP responses to patternreversal stimulation in three carriers, one with particularly positive interocular correlations (A; subject 3 in Table 2) and two with particularly negative correlations ( $\mathbf{B}$; subject 8 in Table 2) and (C; subject 4 in Table 2). Interhemispheric mfVEP difference traces (left) and the respective interocular correlation coefficients (right) are as depicted in Figure 1. The arrows in $\mathbf{B}$ and $\mathbf{C}$ indicate visual field locations with particularly negative correlations between the eyes, which applied to only a few visual field locations.
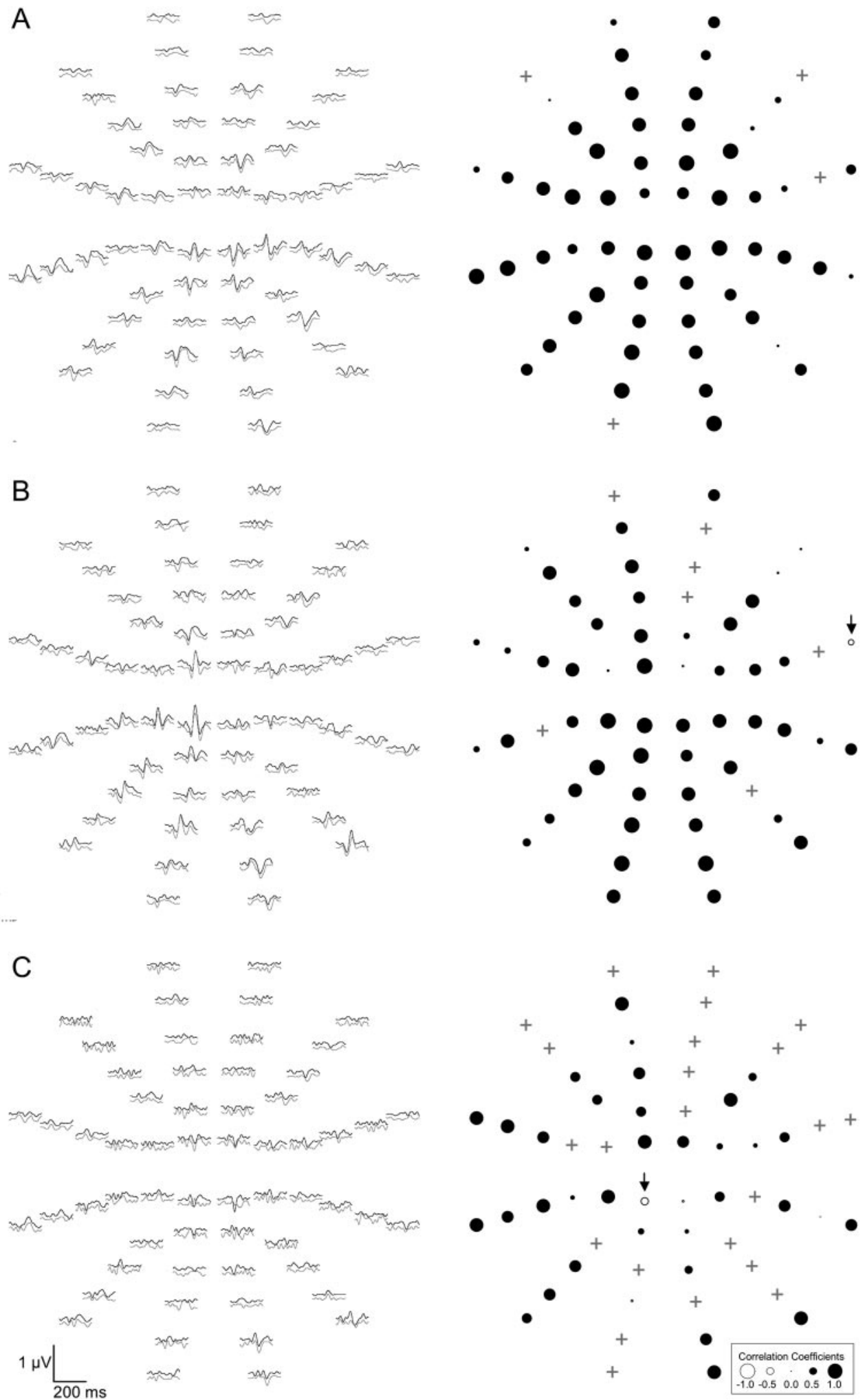

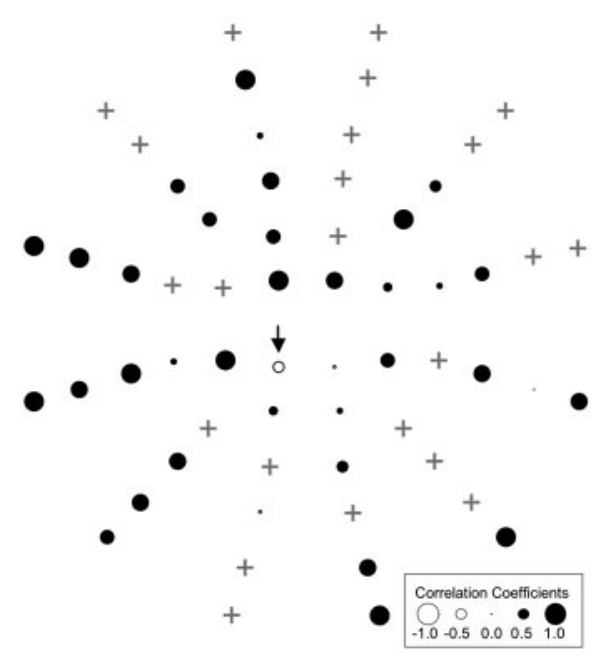

tional VEPs, we did not find a significant deviation from control subjects that would indicate albinotic misrouting. ${ }^{25}$ Although local heterogeneous abnormalities occurring at a retinal extent smaller than the focal stimulus size cannot be fully excluded, clearly no abnormalities of the size reported in feline carriers are evident. Furthermore, in a preceding mfVEP study, we reported normal representations in another type of carrier, that of ocular albinism (OA1). This finding is of particular interest, as these carriers tend to have ocular abnormalities-specifically, local hypopigmentation of fundus and iris. Finally, all preceding conventional VEP studies that used interocular comparisons of response lateralizations to identify misrouted input to the visual cortex failed to reveal response abnormalities in human carriers of albinism. Taken together, no significant representation of abnormalities appears to be evident in human carriers of albinism. 


\section{Entire stimulated visual field $\left( \pm 22^{\circ}\right)$}
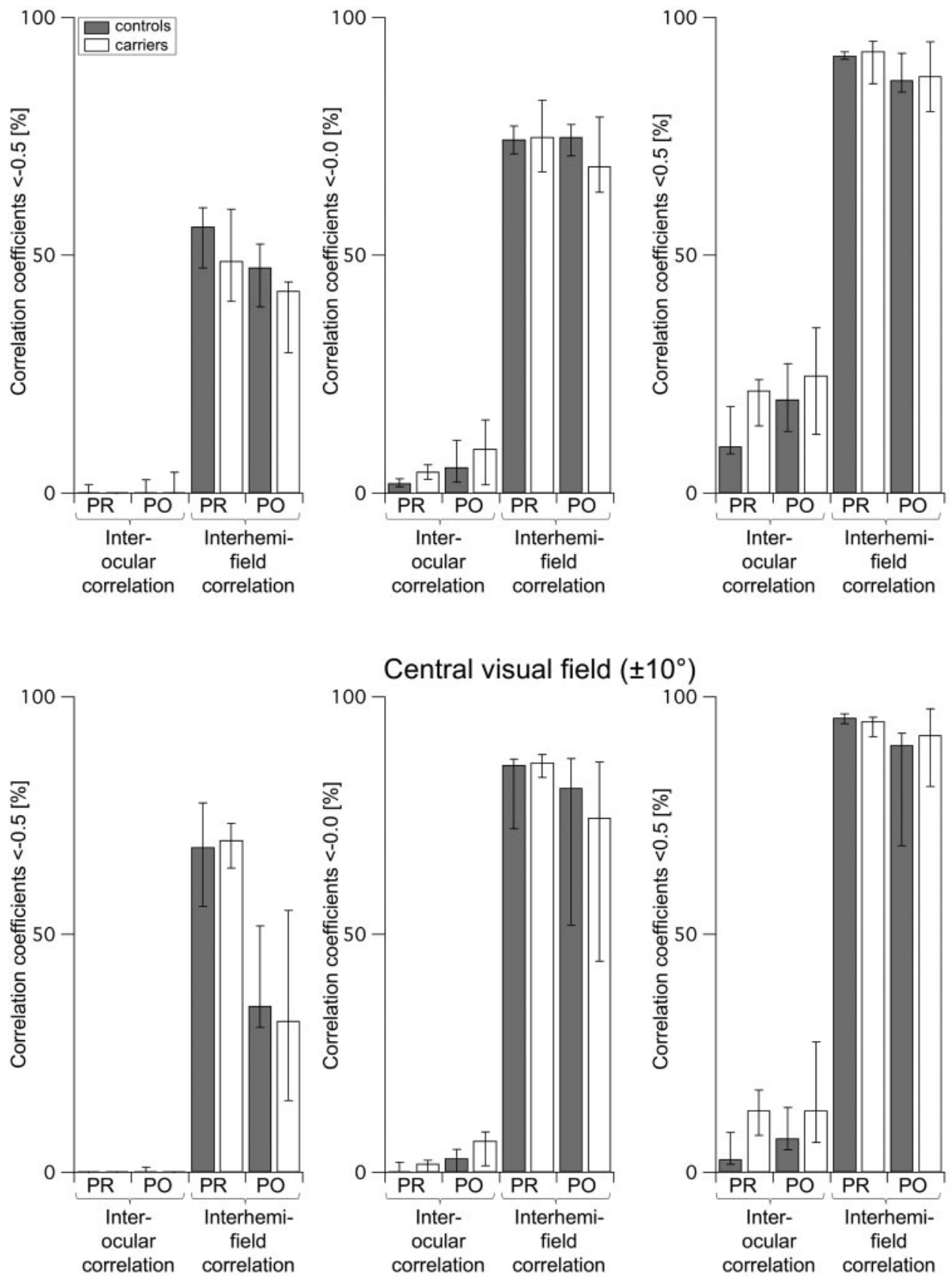

$\left( \pm 10^{\circ}\right)$

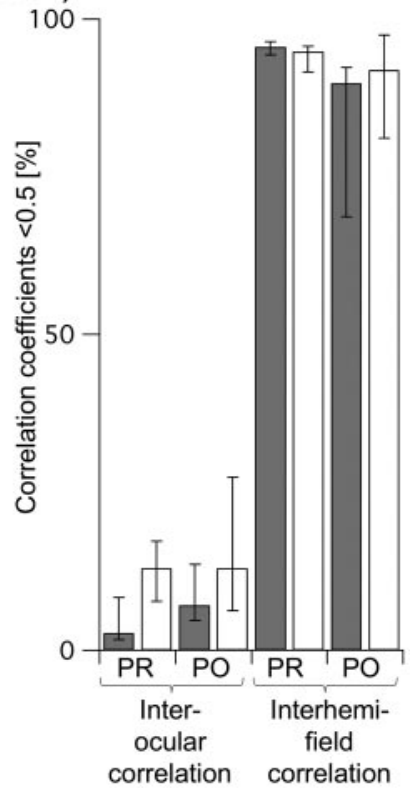

FiguRE 5. Comparison of the frequency of correlation-coefficients < $-0.5,0.0$, and 0.5 (left, middle, and right, respectively) for interocular and interhemifield correlations of responses to pattern-reversal $(\mathrm{PR})$ and pattern-onset (PO) stimulation for controls and carriers. Values are given as the medians and upper and lower quartiles for an analysis of all visual field locations stimulated ( $t o p$ ) and for an analysis confined to the central visual field locations (bottom). No significant differences between the two groups were evident.

\section{Albino Cats as a Model for Human Albinism?}

As humans differ from cats in their absence of representation abnormalities in albinism carriers, the mechanisms behind misrouting of the optic nerves and its consequences for the visual system may also differ. Accordingly, the view that cats can actually serve as a valid model for the effect of albinism in the visual system is debatable. Indeed, organization patterns in the visual cortex of albino cats appear to be more heterogeneous than in humans. The entity of three so-far-described organization patterns has been encountered in cat models of albinism (reviewed in Hoffmann et al. ${ }^{40}$ ), while current evidence in human and nonhuman primates supports the occurrence of only one of these patterns-namely, the true albino pattern. ${ }^{40-43}$ This finding may be associated with differences in the visual systems (e.g., a parallel geniculate input to V1 and
V2 in cats) ${ }^{44}$ that are not evident in humans. In conclusion, although it is not possible to study the visual system in human albinism in the same detail as in animal models, interspecies differences producing diverging evidence are evident from the results of the current and preceding studies. Thus, further discrepancies between the species that may be uncovered in the future would not come as a surprise. Recent advances in noninvasive techniques for a detailed analysis of the neurophysiology of sensory systems directly in humans are therefore particularly beneficial for our understanding of the visual system in human albinism.

\section{Pattern-Reversal versus Pattern-Onset mfVEP}

For conventional VEPs there are lateralization differences of pattern-reversal and -onset responses. ${ }^{45}$ These render pattern 
onset preferable for the detection of response lateralizations with conventional VEPs. In contrast, pattern-reversal and -onset mfVEPs share a high degree of similarity (correlation coefficient, 0.6$)^{46,47}$ and are, as a consequence, likely to originate from similar generators. This view is further supported by the findings of the present study that the distributions of the coefficients for interocular and interhemifield response correlations are similar for pattern-reversal and -onset mfVEPs. For high stimulation rates, as used in the present study, patternreversal stimulation appears to be better suited than patternonset stimulation to sample a great expanse of visual field with mfVEPs for representation abnormalities, as higher SNRs achieved for pattern-reversal in the periphery as detailed in the Methods section and in Hoffmann et al. ${ }^{47}$ It should be noted, however, that for low stimulation rates (sparse stimulation) pattern-onset stimulation might be beneficial as indicated by James et al. ${ }^{46,48}$ and Maddess et al. ${ }^{49}$ Further, mfVEPs to pattern-onset are favorable in patients with nystagmus. ${ }^{50-52}$

Correlation-based interocular comparisons of interhemispheric mfVEP differences allow for the detection of abnormal visual field representations in albinism. In the present study, we used this approach to compare response lateralizations in the visual cortex of controls and of OCA1a carriers. No significant differences were obtained, indicating the lack of representation abnormalities in human OCA1a carriers, which contrasts with previous findings in feline carriers of albinism.

\section{Acknowledgments}

The authors thank the subjects for their cooperation and two anonymous reviewers for helpful comments.

\section{References}

1. Lund RC. Uncrossed visual pathways of hooded and albino rats. Science. 1965;149:1505-1507.

2. Creel DJ. Visual system anomaly associated with albinism in the cat. Nature. 1971;231:465-466.

3. Kaas JH, Guillery RW. The transfer of abnormal visual field representations from the dorsal lateral geniculate nucleus to the visual cortex in Siamese cats. Brain Res. 1973;59:61-95.

4. Guillery RW, Okoro AN, Witkop CJ Jr. Abnormal visual pathways in the brain of a human albino. Brain Res. 1975;96:373-377.

5. Hedera P, Lai S, Haacke EM, et al. Abnormal connectivity of the visual pathways in human albinos demonstrated by susceptibilitysensitized MRI. Neurology. 1994;44:1921-1926.

6. Schmitz B, Kasmann-Kellner B, Schafer T, et al. Monocular visual activation patterns in albinism as revealed by functional magnetic resonance imaging. Hum Brain Mapp. 2004;23:40 -52.

7. Morland AB, Hoffmann MB, Neveu M, Holder GE. Abnormal visual projection in a human albino studied with functional magnetic resonance imaging and visual evoked potentials. J Neurol Neurosurg Psychiatry. 2002;72:523-526.

8. Guillery RW. Neural abnormalities in albinos. Trends Neurosci. 1986;18:364-367.

9. Tremblay F, De Becker I, Cheung C, LaRoche GR. Visual evoked potentials with crossed asymmetry in incomplete congenital stationary night blindness. Invest Opbthalmol Vis Sci. 1996;37:17831792.

10. Ung T, Allen LE, Moore AT, et al. Is optic nerve fibre mis-routing a feature of congenital stationary night blindness? Doc Opbthalmol. 2005; 111:169-178.

11. van Genderen MM, Riemslag FC, Schuil J, Hoeben FP, Stilma JS, Meire FM. Chiasmal misrouting and foveal hypoplasia without albinism. Br J Ophthalmol. 2006;90:1098-1102.

12. Käsmann-Kellner B, Seitz B. Phenotype of the visual system in oculocutaneous and ocular albinism (in German). Ophthalmologe. 2007; 104:648-661.

13. Zuhlke C, Stell A, Kasmann-Kellner B. Genetics of oculocutaneous albinism (in German). Opbthalmologe. 2007;104:674-680.
14. Leventhal AG, Vitek DJ, Creel DJ. Abnormal visual pathways in normally pigmented cats that are heterozygous for albinism. Science. 1985;229:1395-1397.

15. Ault SJ, Leventhal AG, Vitek DJ, Creel DJ. Abnormal ipsilateral visual field representation in areas 17 and 18 of hypopigmented cats. J Comp Neurol. 1995;354:181-192.

16. Hoffmann MB, Schmidtborn LC, Morland AB. Abnormal representations in the visual cortex of patients with albinism: diagnostic aid and model for the investigation of the self-organisation of the visual cortex (in German). Ophthalmologe. 2007;104:666-673.

17. Apkarian P, Reits D, Spekreijse H, van Dorp D. A decisive electrophysiological test for human albinism. Electroencephalogr Clin Neurophysiol. 1983;55:513-531.

18. Castle D, Kromberg J, Kowalsky R, et al. Visual evoked potentials in Negro carriers of the gene for tyrosinase positive oculocutaneous albinism. J Med Genet. 1988;25(12):835-837.

19. Shallo-Hoffmann J, Apkarian P. Visual evoked response asymmetry only in the albino member of a family with congenital nystagmus. Invest Ophthalmol Vis Sci. 1993;34(3):682-689.

20. Apkarian P. Electrodiagnosis in paediatric ophthalmogenetics. Int J Psychophysiol. 1994;16(2-3):229-243.

21. Fitzgerald $\mathrm{K}$, Cibis $\mathrm{CW}$. The value of flash visual evoked potentials in albinism. J Pediatr Ophthalmol Strabismus. 1994;31(1):18-25.

22. Russell-Eggitt I, Kriss A, Taylor DS. Albinism in childhood: a flash VEP and ERG study. Br J Ophthalmol. 1990;74(3):136-140.

23. Rudolph G, Meindl A, Bechmann M, et al. X-linked ocular albinism (Nettleship-Falls): a novel 29-bp deletion in exon 1. Carrier detection by ophthalmic examination and DNA analysis. Graefes Arch Clin Exp Ophthalmol. 2001;239:167-172.

24. Lauronen L, Jalkanen R, Huttunen J, et al. Abnormal crossing of the optic fibres shown by evoked magnetic fields in patients with ocular albinism with a novel mutation in the OA1 gene. $\mathrm{BrJ}$ Opbthalmol. 2005;89(7):820 - 824.

25. Hoffmann MB, Lorenz B, Preising M, Seufert PS. Assessment of cortical visual field representations with multifocal VEPs in control subjects, patients with albinism, and female carriers of ocular albinism. Invest Ophthalmol Vis Sci. 2006;47:3195-3201.

26. Sutter EE. The fast m-transform: a fast computation of cross-correlations with binary m-sequences. SIAM J Comput. 1991;20:686694.

27. Baseler HA, Sutter EE, Klein SA, Carney T. The topography of visual evoked response properties across the visual field. Electroencephalogr Clin Neurophysiol. 1994;90:65-81.

28. Klistorner AI, Graham SL, Grigg JR, Billson FA. Multifocal topographic visual evoked potential: improving objective detection of local visual field defects. Invest Ophthalmol Vis Sci. 1998;39:937950 .

29. Hood DC, Greenstein VC. Multifocal VEP and ganglion cell damage: applications and limitations for the study of glaucoma. Prog Retin Eye Res. 2003;22:201-251.

30. Schmitz B, Schaefer T, Krick CM, Reith W, Backens M, KäsmannKellner B. Configuration of the optic chiasm in humans with albinism as revealed by magnetic resonance imaging. Invest $O p b$ thalmol Vis Sci. 2003;44(1):16-21.

31. Bach M. The Freiburg Visual Acuity Test: automatic measurement of visual acuity. Optom Vis Sci. 1996;73:49-53.

32. Soong F, Levin AV, Westall CA. Comparison of techniques for detecting visually evoked potential asymmetry in albinism. $J$ AAPOS. 2000;4:302-310

33. Pott JW, Jansonius NM, Kooijman AC. Chiasmal coefficient of flash and pattern visual evoked potentials for detection of chiasmal misrouting in albinism. Doc Opbthalmol. 2003;106:137-143.

34. Hoffmann MB, Lorenz B, Morland AB, Schmidtborn LC. Misrouting of the optic nerves in albinism: estimation of the extent with visual evoked potentials. Invest Opbthalmol Vis Sci. 2005;46:38923898.

35. Zhang X, Hood DC, Chen CS, Hong JE. A signal-to-noise analysis of multifocal VEP responses: an objective definition for poor records. Doc Ophthalmol. 2002;104:287-302.

36. Hood DC, Zhang X, Hong JE, Chen CS. Quantifying the benefits of additional channels of multifocal VEP recording. Doc Ophthalmol. 2002; 104:303-320. 
37. Holm S. A simple sequentially rejective multiple test procedure Scand J Stat. 1979;6:65-70.

38. Hood DC, Zhang X, Rodarte C, Yang EB, Ohri N, Fortune B, Johnson CA. Determining abnormal interocular latencies of multifocal visual evoked potentials. Doc Ophthalmol. 2004;109:177-187.

39. Hood DC, Ohri N, Yang EB, et al. Determining abnormal latencies of multifocal visual evoked potentials: a monocular analysis. Doc Ophthalmol. 2004;109:189-199.

40. Hoffmann MB, Tolhurst DJ, Moore AT, Morland AB. Organization of the visual cortex in human albinism. J Neurosci. 2003;23:8921-8930.

41. Guillery RW, Hickey TL, Kaas JH, Felleman DJ, Debruyn EJ, Sparks DL. Abnormal central visual pathways in the brain of an albino green monkey (Cercopithecus aethiops). J Comp Neurol. 1984; 226:165-183.

42. Morland AB, Baseler HA, Hoffmann MB, Sharpe LT, Wandell BA. Abnormal retinotopic representations in human visual cortex revealed by fMRI. Acta Psychol (Amst). 2001;107:229-247.

43. Hoffmann MB, Seufert PS, Schmidtborn LC. Perceptual relevance of abnormal visual field representations: static visual field perimetry in human albinism. Br J Ophthalmol. 2007;91:509-513.

44. Stone J, Dreher B, Leventhal A. Hierarchical and parallel mechanisms in the organization of visual cortex. Brain Res. 1979;180: 345-394.
45. Shawkat FS, Kriss A. A study of the effects of contrast change on pattern VEPS, and the transition between onset, reversal and offset modes of stimulation. Doc Ophthalmol. 2000;101:73-89.

46. James AC. The pattern-pulse multifocal visual evoked potential. Invest Ophthalmol Vis Sci. 2003;44:879-890.

47. Hoffmann MB, Straube S, Bach B. Pattern-onset stimulation boosts central multifocal VEP responses. J Vision. 2003;3:432-439.

48. James AC, Ruseckaite R, Maddess T. Effect of temporal sparseness and dichoptic presentation on multifocal visual evoked potentials. Vis Neurosci. 2005;22:45-54.

49. Maddess T, James AC, Bowman EA. Contrast response of temporally sparse dichoptic multifocal visual evoked potentials. Vis Neurosci. 2005;22:153-162.

50. Hoffmann MB, Seufert PS. Simulated nystagmus reduces patternreversal more strongly than pattern-onset multifocal visual evoked potentials. Clin Neurophysiol. 2005;116:1723-1732.

51. Hoffmann MB, Seufert PS, Bach M. Simulated nystagmus suppresses pattern-reversal but not pattern-onset visual evoked potentials. Clin Neurophysiol. 2004;115:2659-2665.

52. Saunders KJ, Brown G, McCulloch DL. Pattern-onset visual evoked potentials: more useful than reversal for patients with nystagmus. Doc Ophthalmol. 1998;94:265-274. 